\title{
SISTEMA DE NAVEGACIÓN AUTOMÁTICA CONTROLADA POR VOZ PARA UNA SILLA DE RUEDAS
}

\author{
Marcelo Arévalo Lu₹uriaga ${ }^{1}$
}

\begin{abstract}
RESUMEN
EL objetivo principal del presente proyecto fue desarrollar un sistema autónomo capaz de movilizar a personas discapacitadas parapléjicas sobre una silla de ruedas, utilizando un simple comando o instrucción de voz.

El sistema de control del navegador está basado en un microcontrolador tipo PIC 16f877A como elemento maestro del comando y de 2 microcontroladores esclavos para la ejecución del reconocimiento de VOz y manejo de los actuadores. El microcontrolador RCS 300 realiza el almacenamiento y reconocimiento de los comandos de voz utilizados para el control del equipo.

Este equipo posee también la alternativa de un comando manual mediante un joystick ubicado en la parte frontal de la silla, siendo esta función una de las 15 posibilidades de trabajo que posee el equipo, así mismo el sistema está dotado con un conjunto de 6 sensores ultrasónicos programables que entran a funcionar en el modo autónomo y sirven de seguridad al hablante ante posibles colisiones con obstáculos en todo su perímetro y también abismos que encuentre durante su trayectoria.
\end{abstract}

\footnotetext{
${ }^{1}$ Facultad de Ciencias de la Ingeniería, Universidad Tecnológica Equinoccial, Av. Occidental y Mariana de Jesús. Quito, Ecuador.mjarevalo@ute.edu.ec
} 


\section{INTRODUCCIÓN}

El análisis de una señal de voz o acústica inicia desde cuando la señal es generada en las cuerdas vocales del hablante y, se le considera una señal sonora.

Las señales sonoras que se caracterizan por tener alta energía y una frecuencia que va en el rango de los $300 \mathrm{~Hz}$ a $4000 \mathrm{~Hz}$ las cuales se generan por intermedio de las cuerdas vocales y además presentan cierta periodicidad.

La señal de vOz básicamente está constituida por ondas de presión producidas por el aparato humano. La captura de este tipo de señal se realiza mediante un micrófono, el cual se encargará de convertir la onda de presión sonora en una señal eléctrica.

La señal analógica obtenida se debe convertir formato digital, para poder procesarla en la computadora lo que se realiza mediante dos procesos: muestreo y cuantificación. Este proceso se conoce como Modulación por Código de Pulsos (PCM).

Posteriormente se hace un pre procesamiento de la señal vocal. Esto se realiza a través de técnicas, que permiten extraer la información acústica directamente a partir de la señal vocal emitida.

Dentro del reconocimiento de señales acústicas se puede tener tres tipos de sistemas de reconocimiento los cuales son:

- Dependiente del hablante,

- Independiente del hablante, $\mathrm{y}$

- Adaptable al hablante

Un sistema dependiente del hablante es desarrollado para funcionar para un sólo hablante.

Un sistema independiente del hablante es desarrollado para funcionar para cualquier hablante de un determinado tipo (por ejemplo, Inglés Americano).

Un sistema adaptable al hablante es desarrollado para adecuar su funcionamiento a las características de nuevos hablantes. 


\section{METODOLOGÍA}

En el Diseño e implementación del equipo se consideraron tres fases:

- la estructura mecánica sobre la silla de ruedas.

- el hardware.

- y el software.

Para conseguir la movilidad autónoma de la silla fue necesario adaptar engranes a las ruedas posteriores y transmitir movimiento con una cadena desde un motor acoplado a un motor reductor, como se puede apreciar en la figura 1.

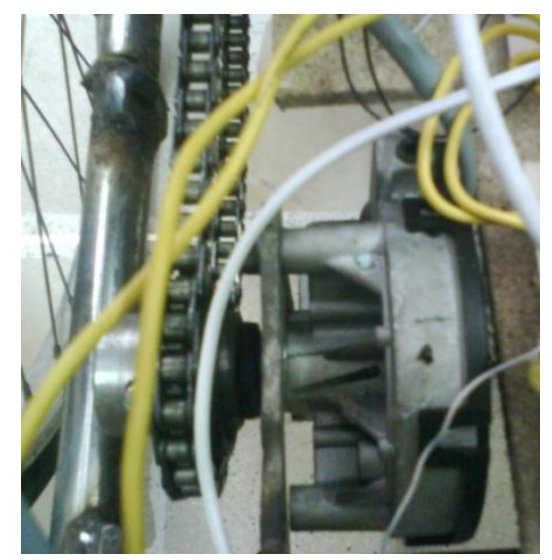

Figura 1. Motor reductor acoplado a eje de la silla

De igual manera se hicieron adaptaciones mecánicas para poder transportar la fuente de poder del conjunto y ubicar estratégicamente los sensores ultrasónicos en la periferia de la silla, figuras 2 y 3. 


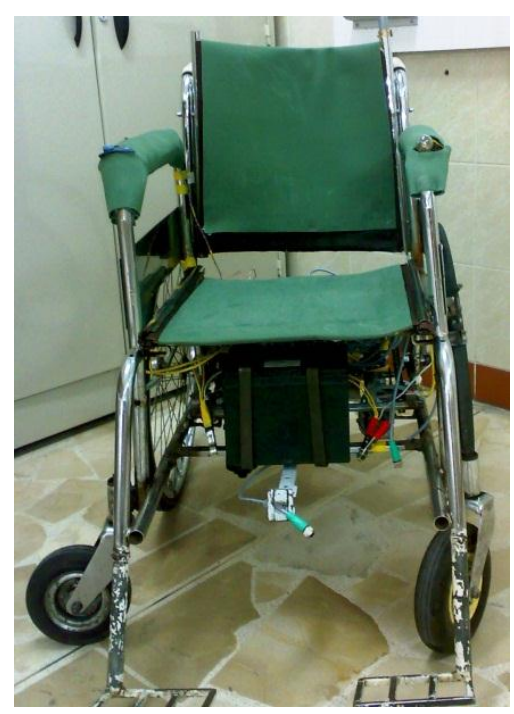

Figura 2. Distribución de sensores en la periferia de la silla

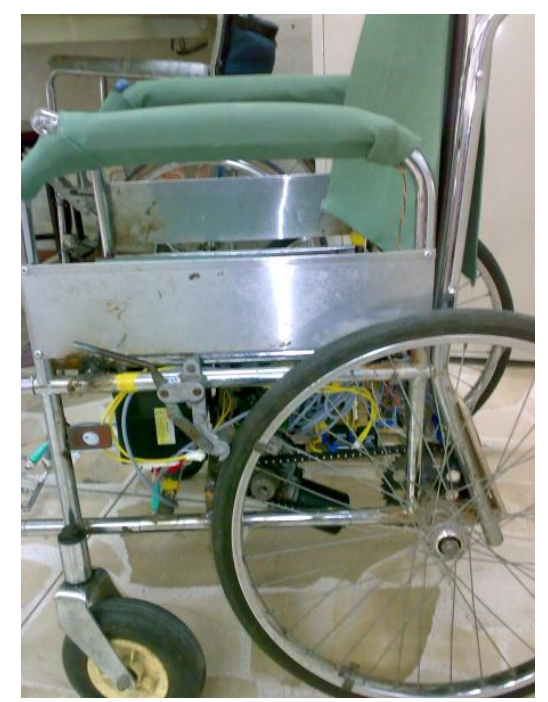

Figura 3. Estructura para parte electrónica.

Toda la estructura está comandada por un conjunto de microcontroladores que constituyen la parte inteligente de la silla, el hardware de este sistema está formado por varios elementos electrónicos unidos en una tarjeta madre que recibirá las señales de los elementos externos y generará el comando lógico de las saldas de control.

Además de la tarjeta madre, figura 4, se tienen dos tarjetas electrónicas enlazadas a esta por un bus de datos, la primera encargada del reconocimiento de la voz, figura 5, cuyo esquema se presenta en la figura 6, y la segunda encargada del manejo de la potencia de la parte motriz, figura 7. 


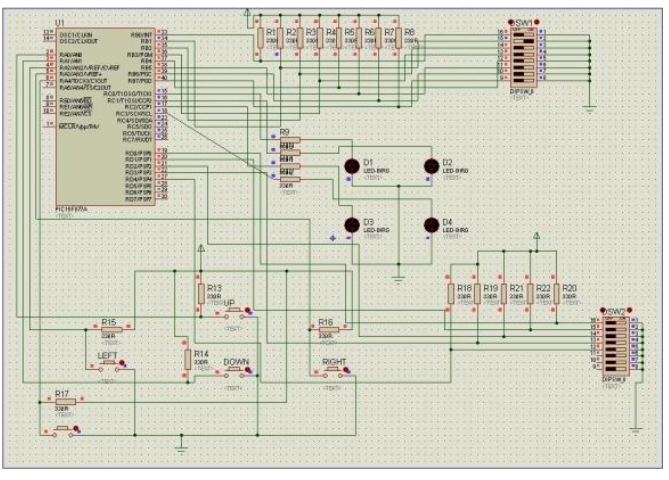

Figura 4. Tarjeta madre de control

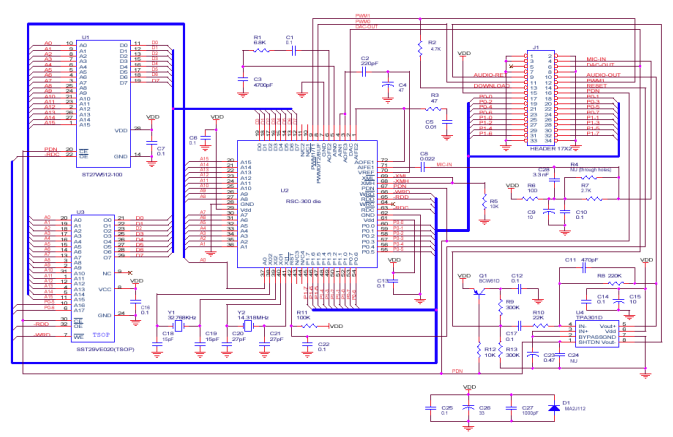

Figura 6. Esquema de la tarjeta de reconocimiento de voz.

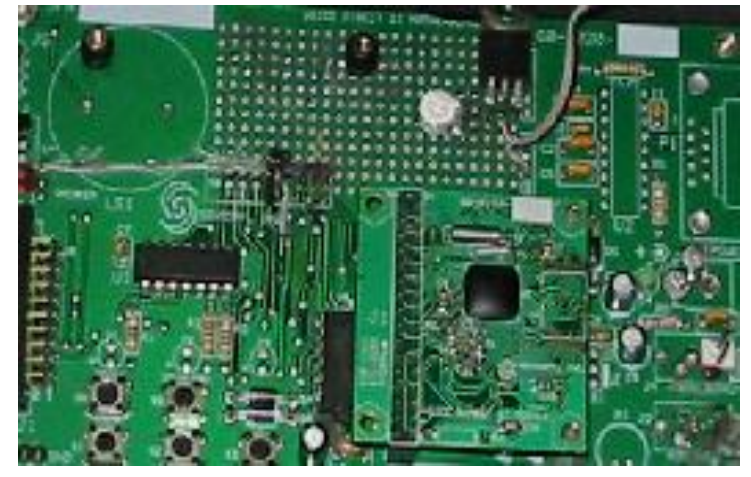

Figura 5. Tarjeta de reconocimiento de voz

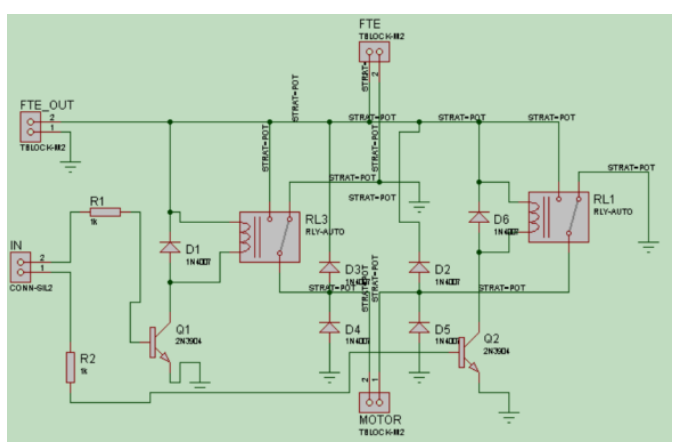

Figura 7. Tarjeta de potencia, parte motriz

Por último el software de programación de los microcontroladores, tanto PIC como RSC 300, se desarrolló en lenguaje ensamblador, compilado y descargado en los mismos hasta obtener los resultados deseados. 


\section{RESULTADOS}

Luego de la aplicación de la metodología descrita, se obtuvo un equipo que tiene la capacidad de realizar las siguientes subrutinas de acción, descritas en la tabla 1.

Tabla 1. Palabras que reconoce el equipo.

\begin{tabular}{ll}
\hline Orden & Descripción \\
\hline Auto & Modo automático \\
Manual & Modo manual \\
Adelante & Movimiento hacia adelante \\
Atrás & Movimiento hacia atrás \\
Izquierda & Giro a la izquierda \\
Derecha & Giro a la derecha \\
Paro & Detiene \\
\hline
\end{tabular}

Como se puede observar en la tabla 1, únicamente se ocupan 7 de las 15 posibles instrucciones que puede recibir el microcontrolador RSC 300 en el sistema dependiente del hablante, dando la posibilidad de aumentar las subrutinas de acción de este equipo, añadiendo accesorios que mejoren el desempeño del equipo.

De igual manera los sensores ultrasónicos son calibrables a una distancia que va desde $5 \mathrm{~cm}$ hasta 80 cm desde el mismo hacia el obstáculo, lo que permite que la silla en modo automático pueda mantener una trayectoria y evadir cualquier tipo de obstáculo o abismo. 


\section{CONCLUSIÓN}

Se ha comprobado que el reconocimiento de voz es una gran herramienta que no ha sido desarrollada en su totalidad aun en nuestro país, y que ofrece una verdadera comunicación directa y confiable entre el hombre y la máquina, cosa que antiguamente se constituía en un sueño, ya que la manera de comunicación con la maquina era por medio de interfaces gráficas, botones o hmi’s que necesitaban de acciones físicas del usuario. Mas ahora se puede controlar a todo tipo de maquinaria por medio de nuestra voz y con palabras comunes como si se estuviera hablando con un operario más dentro de la industria.

Este sistema de exploración posee ventajas sobre los otros sistemas existentes, pues en este caso el usuario no necesita más que su voz para poder realizarlo, permitiendo de esta manera ser manejado por personas físicamente discapacitadas y para nuestro caso, parapléjicas.

Este equipo contribuye a relacionar la parte científica de la Universidad con la comunidad, respondiendo a su responsabilidad social. 


\section{BIBLIOGRAFÍA}

J.M. Montero*, J. Gutiérrez-Arriola*, J. Colás*, J. Macías-Guarasa*, E. Enríquez**, J.M. Pardo*, “desarrollo de un sintetizador de habla en español”, Universidad Politécnica de Madrid, 2008

Hoja de especificaciones técnicas del microcontrolador RSC 300/364 de la sensoryinc 2009

Lleida, E., Rose, R.C.: "Utterance verification in continous speech recognition: decoding and training procedures" IEEE Transactions on Speech and Audio Processing, vol. 8(2), pp. 126--139, 2008.

Xuedong Huang, Alex Acero, Hsiao-Wuen Hon: "Spoken Language Processing: A Guide to Theory, Algorithm and System Development", Prentice Hall, 2009.

http://www.euskalnet.net/iosus/speech/recog.html

Ron Cole. (2007). “Aplicaciones de reconocimiento de voz”, Oregon Institute. 